\title{
Definition of retrosternal thyroid growth
}

\author{
Ernst G. Eising ${ }^{1}$, Sandra Rosenbaum-Krumme ${ }^{2}$, Walter Jentzen ${ }^{2}$, Alfred Gödecke $^{1}$, Andreas Bockisch ${ }^{2}$ \\ ${ }^{1}$ Radprax, Arnsberg, Germany \\ ${ }^{2}$ Clinic for Nuclear Medicine, University of Duisburg-Essen, Duisburg and Essen, Germany \\ Email: e-g-e@web.de
}

Received 19 March 2012; revised 23 April 2012; accepted 26 May 2012

\section{ABSTRACT}

The diagnosis of a retrosternal thyroid growth (RTG) causes extended surgical exploration and a different surgical treatment planning (partial sternotomy, thoracotomy) and is usually made by the help of thyroid scintigraphy and ultrasonography. But both examinations have problems in determining the retrosternal thyroid extend in the complex anatomy of sternal bone and often pathologically altered manubrioclavicular joints (MCJ). This study evaluates the variation of anatomical structures in the upper sternal region, the position of the sternal markers during scintigraphy, and the frequency of enhanced bone metabolism of the MCJs. For this aim, the positions of the upper edges of the MCJs were measured by X-ray fluoroscopy in 50 consecutive patients. To prove the exactness of an external marker as used in thyroid scintigraphy, the variation of the marker position was also determined. The activity in the MCJs was measured semiquantitatively with bone scintigraphy in further 100 patients (mean age: $62.3 \mathrm{yrs}$, SD: $14.2 \mathrm{yrs}$ ). As results, the upper edges of the medial clavicular edges exceed the upper margin of the sternal edge up to $2.7 \mathrm{~cm}$. The distance between the medial clavicular edges ranged from $2.3-5.6 \mathrm{~cm}$. The position of the sternal marker was correct in the horizontal deviation (mean: $0.1 \mathrm{~cm}$, SD: $0.48 \mathrm{~cm}$ ) but too high in the vertical position (mean: $2.2 \mathrm{~cm}$, SD: $0.67 \mathrm{~cm}$ ). During bone scintigraphy, the MCJs showed no enhanced activitity in $75 / 200$ joints, medium activity in $96 / 200$ joints, and strong enhanced activity in $29 / 200$ joints. In consequence, a high variability in the position of the anatomical structures has to be considered together with a high amount of degenerative alterations. The position of the sternal marker was inappropriate in the vertical direction and overestimated a possible retrosternal growth.

Keywords: Thyroid Gland; Thyroid Scintigraphy; Manubrioclavicular Joints

\section{INTRODUCTION}

The diagnosis of a retrosternal thyroid growth (RTG) can cause extended surgical exploration (sternotomy) and is usually made by thyroid scintigraphy and ultrasonography. But both examinations have problems in determining the retrosternal thyroid extend in the complex anatomy of sternal bone and often pathologically altered manubrioclavicular joints (MCJ). Furthermore, exact criteria for RTG are missing up to now.

Aim of this study was to evaluate the variation in the anatomy of the upper sternal region, the determination of precision of sternal markers as used during thyroid scintigraphy, and the discussion guidelines for the description of intrathoracic thyroid growth on CT. The findings of all these preoperative imaging procedures (including ultrasound) should match to give precise data for surgical treatment planning.

\section{MATERIAL AND METHODS}

\subsection{Patients}

The position of the upper edges of the MCJs was measured by X-ray fluoroscopy in 18 consecutive patients ( 9 female, 9 male, age: 25 - 88 yrs, mean: 56.8 yrs, SD: 21.4 yrs).

The activity in the MCJs was measured semi-quantitatively with bone scintigraphy in further 100 patients (54 female, 46 male, age 15 - 87 yrs, mean: 62.3 yrs SD: 14.16 yrs). 57 of the patients had cancer in their history (24 prostate cancer, 20 breast cancer, 13 other). Patients with evidence of cancer in the MCJs were excluded from the study.

\subsection{Methods}

To prove the exactness of an external marker as used in thyroid scintigraphy, the variation of the marker position was determined by fluoroscopy. As equipment, a Siemens Axiom Iconos MD was used (Siemens Inc., Erlangen, Germany). A lead globule with a defined diameter was used as marker. Because the measured distances depend on distance to the image intensifier and the cho- 
sen magnification, all distances have to be calibrated upon an unique level. The cranial excess of each clavicle compared to the upper sternal edge was assessed as the distance between the medial margins of the clavicles (Figure 1(a)). The position of the lead globule was determined in both axes (vertical compared to the upper margin of the sternal bone and lateral compared to the point of half-distance between the clavicles and not the thoracic spine, because slight rotation of the patients may cause severe variations in horizontal projection of the lead globule (Figure 1(b)).

The scintigraphic measurements were obtained 3 hours after administration of 500 - 600 MBq Tc99m-HDP using the following scale:

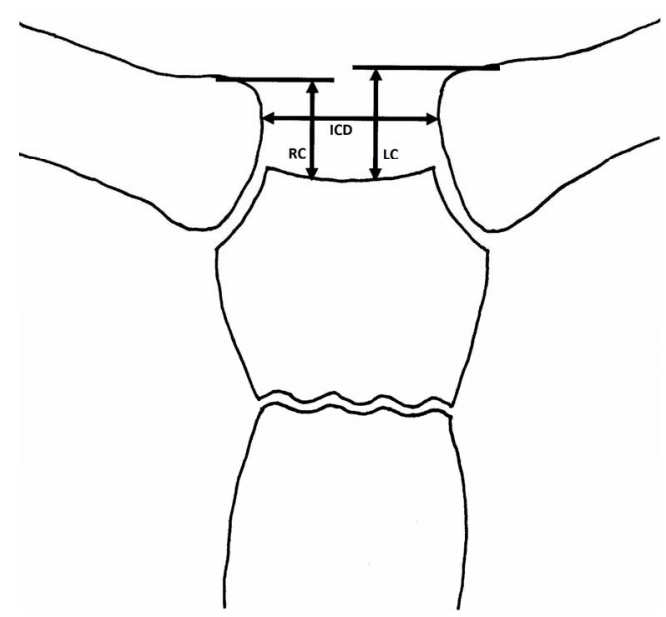

(a)

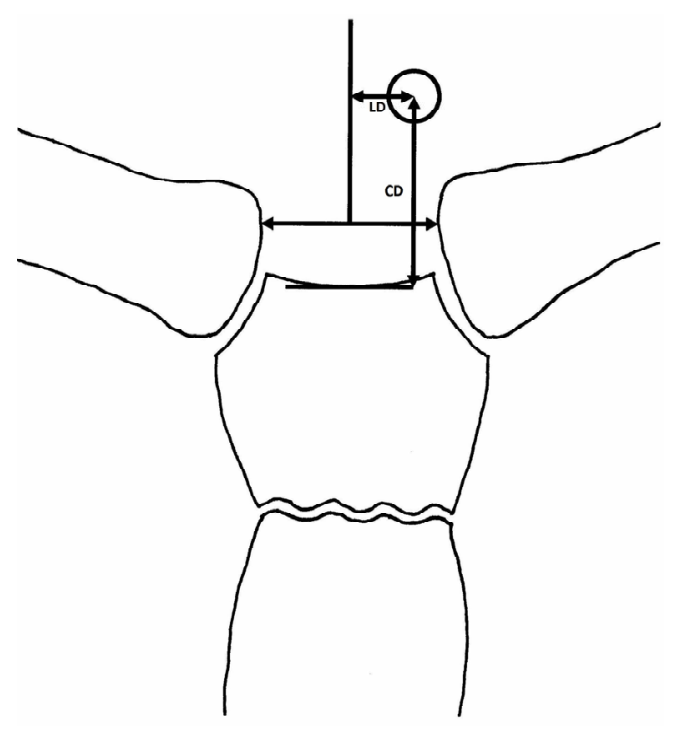

(b)

Figure 1. Schematic drawing of the measurements in the MCJs. (a) Distances (interclavicular distance (ICD), cranial offset of the right (RC) and left (LC) clavicular ending; (b) Marker position (cranial and lateral deviation: $\mathrm{CD}, \mathrm{LD})$.
0: Normal uptake in the MCJ,

1: Slight elevated uptake, and

2: Strong elevated uptake.

As equipment, a double-headed Siemens E-Cam gamma camera was used (Siemens Inc., Erlangen, Germany).

The statistical evaluation was made using descriptive calculations with Excel (Microsoft Inc., Redmond, USA).

\section{RESULTS}

\subsection{Anatomic Variations}

As stated in Table 1, the upper clavicular edges exceeded the upper edge of the manubrial bone by $2 \mathrm{~cm}$. Therefore it is necessary to define, what is meant when stating "retrosternal growth": "retrosternal” in its strict sense or "retroclavicular".

Furthermore, the mean distance between the medial clavicular margins amounted to $4 \mathrm{~cm}$ : a distance, which is smaller than the width of a thyroid gland. So nearly all goiters, whose lower edges reached the level of the manubrium, are covered by the MCJs on both sides.

\subsection{Position of the Sternal Marker}

The measured variation of the sternal marker is given in Table 2. The horizontal deviation scattered around the midline, whereas the vertical deviation had a mean of 2.2 $\mathrm{cm}$, which corresponded to the upper level of the clavicular edges. Assuming a comparable position of the marker during thyroid scintigraphy, an extension of tissue with Tc99m uptake below the marker up to about 2 cm can only be judged as retroclavicular but not as retrosternal.

\subsection{Results of Bone Metabolism in the MCJs}

A pathological elevated activity of the MCJs could be registered in patients exceeding an age of 30 yrs (Table

Table 1. Anatomy of the MCJs (elevation and clavicular distances in $\mathrm{mm}$ ).

\begin{tabular}{cccccc}
\hline Elev. & Min. & Mean & Median & Std.-Dev. & Max. \\
\hline $\begin{array}{c}\text { Right } \\
\text { MCJ }\end{array}$ & 0.93 & 1.74 & 1.63 & 0.51 & 2.70 \\
Left & 1.19 & 1.80 & 1.72 & 0.40 & 2.65 \\
MCJ & & & & & \\
Clav. & 2.25 & 3.91 & 3.88 & 0.72 & 5.56 \\
Dist. & & & & & \\
\hline
\end{tabular}

Table 2. Exactness of the external marker position.

\begin{tabular}{cccccc}
\hline cm & Min. & Mean & Median & Std.-Dev. & Max. \\
\hline Horizontal & -1.16 & -0.10 & 0.00 & 0.48 & 1.13 \\
Vertical & 1.09 & 2.21 & 2.12 & 0.67 & 4.14 \\
\hline
\end{tabular}


Table 3. Scintigraphic activities of the MCJ.

\begin{tabular}{ccccc}
\hline$N$ Age & Grade 0 & Grade 1 & Grade 2 & Sum \\
\hline$<30$ yrs & 4 & 0 & 0 & 4 \\
$30-39$ yrs & 1 & 11 & 0 & 12 \\
$40-49$ yrs & 3 & 17 & 2 & 22 \\
$50-59$ yrs & 17 & 24 & 1 & 42 \\
$60-69$ yrs & 23 & 19 & 8 & 50 \\
$70-79$ yrs & 15 & 25 & 16 & 56 \\
$80-89$ yrs & 8 & 4 & 2 & 14 \\
Sum & 71 & 100 & 29 & 200 \\
& & & & \\
Right MCJ & 28 & 54 & 18 & 100 \\
Left MCJ & 47 & 42 & 11 & 100 \\
Sum & 75 & 96 & 29 & 200 \\
\hline
\end{tabular}

3: upper part). A higher increase in uptake was observed in the groups exceeding 60 yrs. As expected, pathological alterations could be noticed mostly on the dominant right side (Table 3 : lower part).

\section{DISCUSSION}

\subsection{Anatomy}

The definition of thyroid location is not unique: Ignjatovic [1] classified goiters into cervical, retrosternal, substernal, and intrathoracic ones. Batori [2] did not make differences between substernal and retrosternal goiters and defined them with an extent of $>3 \mathrm{~cm}$ beyond the superior thoracic strait. Moron [3] defined retrosternal goiters with at least $50 \%$ of the thyroid below the thyroid inlet. Hedayati [4] defined substernal goiters below the thoracic inlet. In a meta-analysis from Huins from 2008 [5], a new classification has been proposed depending on the relation of the intrathoracic thyroid tissue to the aortic arch and the right atrium. Chauhan [6] stated intrathoracic involvement mostly on the left side (left-to-right ratio was 3:2).

\subsection{Surgery}

Chauhan [6] further reported that a sternotomy was necessary in none of 199 patients with retrosternal goiters. Batori [2] proposed cervicotomy for patients with anterior and superior retrosternal thyroid growth only. Cohen [7] performed sternotomy in cases with malignancies, growth into the posterior mediastinum and especially in cases with ectopic nodules and tissue below the aortic arch. Cui [8], Hardy [9], and Hsu [10] performed cervicotomy in the majority of patients with retrosternal thyroid growth. Alifano [11] emphasized the value of limited upper sternotomy in patients with thymomas and retrosternal goiters. Shpitzer [12] recommended median partial sternotomy in patients with retrosternal goiter and thyroid malignancies as in cases with parathyroid pathologies. Topcu [13] proposed partial sternotomy in patients with intrathoracic goiters of at least $10 \mathrm{~cm}$ di- ameter and extension below the aortic arch. Shah [14] published a case report with a large intrathyreoidal mass to describe the diagnostic and therapeutic dilemma in this tumor location.

The rate of complication was not elevated in patients after thoracotomy compared to those with cervicotomy according to a study of Ignjatovic [1], whereas Huins [5] had found a clear and significant association between the intrathoracic thyroid extent and reported complications.

\subsection{Imaging}

In his early publication from 1985, Pfannenstiel [15] had stressed the value of thoracic computed tomography and ultrasonography in thyroid imaging, especially the impact of CT in diagnosis of intrathoracical masses. Cooper [16] and Ben Nun [17] have confirmed the important role of thoracic CT in subsequent studies.

Thyroid scintigraphy with Tc99m-Pertechnetate indicated in a study of Arnold [18] a similar tracer distribution in 66/85 cases and focal enhanced areas in the Tc99m-Pertechnetate images in 11/19 cases but a slightly better contrast in the I123-images. To achieve a better discrimination of thyroid and non-thyroid uptake, Aydin [19] recommended an additional scan after potassium perchlorate. The value of thyroid scintigraphy has been reported as limited: Varcus [20] stated that the scintigraphic results were worthwhile in $16.7 \%$ (47/369) of the patients with low levels of thyroid stimulating hormone, ectopic thyroid or retrosternal goiter. Tindall [21] found a value of thyroid scintigraphy only in detection of autonomous nodules. Eising [22] reported a possible impact of calcified structures (especially bones) on thyroid scintigraphy.

\subsection{Findings of This Study}

This study demonstrates, that the position of the sternal marker is misleading: "retrosternal" means "retroclavicular” up to an retroclavicular extension of $2.2 \mathrm{~cm}$. So the presence of a retrosternal goiter with consequences on thyroid surgery should only be stated in extensive cases and than by help of computed tomography. These findings match our own experience [22], that a little retrosternal extent of the thyroid gland (demonstrated on CT) is quite normal in elderly subjects and should not be described as pathological.

The high amount of age-dependant pathologic alterations during bone scan indicates, that a change of the anatomical positions has to be expected in the majority of elder patients with possible consequences for surgical intervention.

\section{CONCLUSIONS}

The knowledge of variation in anatomy and the precision 
of sternal markers are important to minimize the errors in determination of retrosternal thyroid growth. As a retrosternal goiter cannot be excluded by scintigraphy in case of missing uptake (e.g., cold nodules), ultrasonography is mandatory additional to thyroid scan.

In cases of relevant intrathoracic masses, a CT has to be performed to describe the exact retrosternal extent together with the relation to anatomic structures, as the aortic arch and possible tumour components in the upper posterior mediastinum.

The development of an unique description of the retrosternal thyroid extent and the according surgical therapeutic approaches should be make in the next time by the scientific surgical societies.

\section{REFERENCES}

[1] Ignjatovic, M., Stanic, V., Cuk, V. and Kostic, Z. (2002) Intrathoracic goiter: Analysis of 21 cases. Acta Chirurgica Jugoslavica, 49, 15-25. doi:10.2298/ACI0201015I

[2] Batori, M., Chatelou, E. and Straniero, A. (2007) Surgical treatment of retrosternal goiter. European Review for Medical and Pharmacological Sciences, 11, 265-268.

[3] Moron, J.C., Singer, J.A. and Sardi, A. (1998) Retrosternal goiter: A six-year institutional review. The American Surgeon, 64, 889-893.

[4] Hedayati, N. and McHenry, C.R. (2002) The clinical presentation and operative management of nodular and diffuse substernal thyroid disease. The American Surgeon, 68, 245-252.

[5] Huins, C.T., Georgalas, C., Mehrzad, H. and Tolley, N.S. (2008) A new classification system for retrosternal goitre based on a systematic review of its complications and management. International Journal of Surgery, 6, 71-76. doi:10.1016/j.ijsu.2007.02.003

[6] Chauhan, A. and Serpell, J.W. (2006) Thyreoidectomy is safe and effective for retrosternal goitre. ANZ Journal of Surgery, 76, 238-242. doi:10.1111/j.1445-2197.2006.03699.x

[7] Cohen, J.P. (2009) Substernal goiters and sternotomy. Laryngoscope, 119, 683-688. doi:10.1002/lary.20102

[8] Cui, Y., Zhang, Z., Li, S., Li, L., Zhang, H. and Li, Z. (2002) Diagnosis and surgical management for retrosternal thyroid mass. Chinese Medical Sciences Journal, 17, 173-177.

[9] Hardy, R.G., Bliss, R.D., Lennard, T.W., Balasubramanian, S.P. and Harrison, B.J. (2009) Management of retrosternal goitres. Annals of the Royal College of Surgeons of England, 91, 8-11. doi:10.1308/003588409X359196

[10] Hsu, B., Reeve, T.S., Guinea, A.I., Robinson, B. and
Delbridge, L. (1996) Recurrent substernal nodular goiter: Incidence and management. Surgery, 120, 1072-1075. doi:10.1016/S0039-6060(96)80057-2

[11] Alifano, M., Parri, S.N., Arab, W.A., Bonfanti, B., Lacava, N., Porello, C. and Boaron, M. (2008) Limited upper sternotomy in general thoracic surgery. Surgery Today, 38, 300-304. doi:10.1007/s00595-007-3626-1

[12] Shpitzer, T., Saute, M., Gilat, H., Rayeh, E., Koren, I., Shvero, J., Bahar, G. and Feinmesser, R. (2007) Adaptation of median partial sternotomy in head and neck surgery. The American Surgeon, 73, 1275-1278.

[13] Topcu, S., Liman, S.T., Canturk, Z., Utkan, Z., Canturk, Z., Corak, S. and Cetinarslan, B. (2008) Necessity for additional incisions with the cervical collar incision to remove retrosternal goiters. Surgery Today, 38, 1072-1077. doi:10.1007/s00595-008-3768-9

[14] Shah, P.J., Bright, T., Singh, S.S., Lang, C.M., Pyragius, M.D., Malycha, P. and Edwards, J.R. (2006) Large retrosternal goitre: A diagnostic and management dilemma. Heart Lung and Circulation, 15, 151-152. doi:10.1016/j.hlc.2005.10.011

[15] Pfannenstiel, P. and Baew-Christow, T. (1985) Roentgen computer tomography and sonography in thyroid gland diagnosis. Zentralblatt fur Chirurgie, 110, 1105-1114.

[16] Cooper, J.C., Nakielny, R. and Talbot, C.H. (1991) The use of computed tomography in the evaluation of large multinodular goitres. Annals of the Royal College of Surgeons of England, 73, 32-35.

[17] Ben Nun, A., Soudack, M. and Best, L.A. (2006) Retrosternal thyroid goiter: 15 years experience. Israel Medical Association Journal, 8, 106-109.

[18] Arnold, J.E. and Pinsky, S. (1976) Comparison of 99mTc and 123I for thyroid imaging. Journal of Nuclear Medicine, 17, 261-267.

[19] Aydin, M., Emri, S. and Caner, B. (2000) Tc-99m pertechnetate scintigraphy before and after potassium perchlorate administration for the diagnosis of retrosternal goiter. Clinical Nuclear Medicine, 25, 467-468. doi:10.1097/00003072-200006000-00015

[20] Varcus, F., Peix, J.L., Berger, N. and Bordos, D. (2002) Thyroid scintigraphy: Its place in preoperative diagnosis of thyroid nodules? Annals of Chirurgie, 127, 685-689. doi:10.1016/S0003-3944(02)00864-7

[21] Tindall, H., Griffiths, A.P. and Penn, N.D. (1987) Is the current use of thyroid scintigraphy rational? Postgraduate Medical Journal, 63, 869-871. doi:10.1136/pgmj.63.744.869

[22] Eising, E.G. and Jentzen, W. (2010) Calcification-related absorption in thyroid scintigraphy. Nuklearmedizin, 49, 13-18. doi:10.3413/nukmed-0204 